Article

\title{
Geographical Distribution of $\beta$-Lactam Resistance among Klebsiella spp. from Selected Health Facilities in Ghana
}

\author{
Elvis Quansah ${ }^{1,3}{ }^{\oplus}$, Prince Amoah Barnie ${ }^{2}{ }^{-}$, Desmond Omane Acheampong ${ }^{3}$, \\ Dorcas Obiri-Yeboah ${ }^{1}$, Richael Odarkor Mills ${ }^{3}$, Ebenezer Asmah ${ }^{1}$, Obed Cudjoe ${ }^{1}$ \\ and Isaac Dadzie ${ }^{4, *}$ \\ 1 Department of Microbiology and Immunology, School of Medical Sciences, University of Cape Coast, \\ Cape Coast, Ghana \\ 2 Department of Forensic Sciences, School of Biological Sciences, University of Cape Coast, Cape Coast, Ghana \\ 3 Department of Biomedical Sciences, School of Allied Health Sciences, University of Cape Coast, \\ Cape Coast, Ghana \\ 4 Department of Medical Laboratory Sciences, School of Allied Health Sciences, University of Cape Coast, \\ Cape Coast, Ghana \\ * Correspondence: idadzie@ucc.edu.gh; Tel.: +2-335-4653-3301
}

Received: 4 July 2019; Accepted: 14 August 2019; Published: 3 September 2019

\begin{abstract}
Lactam-resistant Klebsiella isolates continue to cause multidrug resistance infections worldwide. This study aimed to describe the geographical distribution of extended spectrum $\beta$-lactamase (ESBL), AmpC $\beta$-lactamase (AmpC), and carbapenemase production among 139 Klebsiella isolates recovered from patients at major referral health facilities in Ghana. The phenotypic methods of combined disc diffusion test, modified three-dimensional test, modified Hodge test (MHT), and combined disc test were performed for each isolate to detect ESBL, AmpC, carbapenemase, and metallo- $\beta$-lactamase (MBL) producers, respectively. Except for MBL, all other $\beta$-lactam resistance mechanisms were highest in the healthcare facilities situated in the northern belt of Ghana. Significant regional difference of ESBL producers was observed between the northern and middle belts as well as the northern and southern belts. Genotypic detection with polymerase chain reaction (PCR) revealed the presence of bla TEM 36/139 (25.9\%), bla SHV 40/139 (28.8\%), bla CTX-M 37/139 (26.6\%), bla OXA-48 3/139 (2.16\%), and bla NDM 1/139 (0.72\%) genotypes. In conclusion, there were variations in $\beta$-lactam resistance among Klebsiella spp. from health facilities situated in the northern, middle, and southern belts of Ghana. The study provides preliminary evidence that emphasizes the need to direct more attention to antimicrobial resistance control, especially in the northern belt of Ghana. Findings from this study may be critical for creating and fine-tuning effective antimicrobial resistance control strategies and for informing accurate antibiotic prescription by practitioners.
\end{abstract}

Keywords: $\beta$-lactamases; Klebsiella spp.; extended spectrum beta-lactamase; AmpC; carbapenemase; metallo- $\beta$-lactamase

\section{Introduction}

Among the human infections caused by the genus Klebsiella, Klebsiella pneumoniae remain the most clinically relevant species, accounting for over $70 \%$ of these infections [1]. Also, recent evidence indicates increasing relevance of Klebsiella oxytoca in nosocomial infections [2]. In major referral health facilities in Ghana, K. pneumoniae is among the top three most frequently isolated Gram-negative bacteria that are primarily recovered from patients with urinary tract infection, respiratory tract infection, wound infections, and sepsis [3-5]. 
The emergence of $\beta$-lactamases, including extended spectrum $\beta$-lactamases (ESBLs), AmpC- $\beta$-lactamases (AmpCs), and carbapenemases, particularly in Klebsiella spp., is a central health concern [6]. Until the 1990s, K. pneumoniae typically produced a single or rarely two plasmid-encoded $\beta$-lactamases. Today, K. pneumoniae harboring more than five $\beta$-lactamases are commonplace in some clinical facilities [7]. K. pneumonia is now recognized as "a pool of potent $\beta$-lactamases" [8] and an indicator species for plasmid-encoding $\beta$-lactamases [8]. These $\beta$-lactamases constitute the chief resistance mechanisms that mediate the inactivation of $\beta$-lactam antibiotics by hydrolyzing the amide bond of the $\beta$-lactam ring $[9,10]$. Of all carbapenemases, metallo- $\beta$-lactamase (MBL) are the most transmissible and are particularly troublesome owing to their recent global dissemination and enhanced ability to hydrolyze a wide range of $\beta$-lactams [11].

Recent findings in neighboring West African countries have indicated worrying levels of ESBLs in Klebsiella isolates relative to other Enterobacteriaceae [12,13]. More worrying is the recent increasing documentation of high levels of carbapenemase-encoding genes in K. pneumoniae in a number of African countries [14-16]. Although a modest number of studies have documented high levels of ESBL production and AmpC production in Klebsiella isolates in Ghana [17-20], studies directed at the exploration of carbapenemase production among Klebsiella spp. is very limited.

The northern, middle, and southern belts of Ghana markedly differ in terms of temperature and socioeconomic status [21,22]. Temperature and socioeconomic status are posited to be key determinants of antimicrobial resistance $[23,24]$. Nonetheless, precise data on the regional distribution of these $\beta$-lactam resistance mechanisms among Klebsiella isolates in Ghana seems lacking, preventing the identification of key areas that may need very robust antimicrobial resistance control strategies. Against this background, this study aimed to describe the geographical distribution of ESBL-, AmpC-, and carbapenemase-producing Klebsiella isolates from major referral health centers situated in the northern, middle, and southern belts of Ghana using phenotypic and genotypic techniques. The results from this study will provide a preliminary local evidence of regions in Ghana that may need robust antimicrobial resistance control strategies.

\section{Materials and Method}

\subsection{Collection and Identification of Isolates}

Nonrepetitive isolates were collected from October 2017 to March 2018. To ensure fair representation, healthcare facilities that serve as referral centers in the northern, middle, and southern belts of Ghana were selected for the study. Klebsiella isolates were obtained from Tamale Teaching Hospital and Bolgatanga Regional Hospital (northern belt), Komfo Anokye Teaching Hospital (middle belt) and Cape Coast Teaching Hospital and Effia Nkwanta Regional Hospital (southern belt). These isolates were transported in Luria-Bertani (LB) broth (Oxoid, Basingstoke Hampshire, UK) to the clinical microbiology laboratory (Department of Biomedical Science, University of Cape Coast, Cape Coast, Ghana) for investigation. Isolates were subcultured on McConkey agar media (Oxoid, Basingstoke Hampshire, UK). All the isolates were reidentified using Gram staining and a panel of biochemical tests [25].

\subsection{Phenotypic Techniques}

Antimicrobial Susceptibility Testing (AST)

Antimicrobial susceptibility testing (AST) was carried out on Mueller-Hinton agar (Techno Pharm Chem, Delhi, India) and interpreted according to the Clinical and Laboratory Standards Institute (CLSI) guidelines [26]. The antimicrobial susceptibility testing was performed using ampicillin $(10 \mu \mathrm{g})$, tetracycline $(10 \mu \mathrm{g})$, cotrimoxazole $(25 \mu \mathrm{g})$, gentamicin $(10 \mu \mathrm{g})$, cefuroxime $(30 \mu \mathrm{g})$, chloramphenicol $(10 \mu \mathrm{g})$, ceftriaxone $(30 \mu \mathrm{g})$, cefotaxime $(30 \mu \mathrm{g})$, ciprofloxacin $(5 \mu \mathrm{g})$, amikacin $(30 \mu \mathrm{g})$, meropenem 
$(10 \mu \mathrm{g})$, imipenem $(10 \mu \mathrm{g})$, ertapenem $(10 \mu \mathrm{g})$, cefoxitin $(30 \mu \mathrm{g})$, and cefotetan $(30 \mu \mathrm{g})$ (BIO-RAD, Munich, Germany). Escherichia Coli ATCC 25299 was used as a quality control strain.

Detection of ESBL production was carried out on a Mueller-Hinton agar (Oxoid, Basingstoke Hampshire, UK) plate seeded with the test isolate. Combined discs of either cefotaxime $(30 \mu \mathrm{g}) /$ clavulanic acid $(10 \mu \mathrm{g})$ or ceftazidime $(30 \mu \mathrm{g}) /$ clavulanic acid $(10 \mu \mathrm{g})$ (BIO-RAD, Munich, Germany) was placed $20 \mathrm{~mm}$ away from single discs of cefotaxime $(30 \mu \mathrm{g})$ or ceftazidime $(30 \mu \mathrm{g})$, respectively, and incubated at $37^{\circ} \mathrm{C}$ overnight. An enhanced zone of inhibition $(>5 \mathrm{~mm})$ around the combined disc relative to the single disc was considered positive for the production of ESBL [27]. Escherichia Coli ATCC 25299 was used as a negative control strain, whereas K. pneumoniae ATCC 700603 were used as positive control.

Isolates that were resistant to either cefoxitin $(30 \mu \mathrm{g})$, cefotetan $(30 \mu \mathrm{g})$, or both after antimicrobial susceptibility screening were selected for confirmation of AmpC production using modified three-dimension test. The procedure described by Deotale et al. [28] was used. The positive test was read as a small heart-shaped indentation toward the cefoxitin or cefotetan disc seen at the junction of the slit along the line of inhibition.

Isolates resistant to the carbapenem-based drugs were screened for carbapenemase production using modified Hodge test, as recommended by CLSI [26]. A total of four organisms were tested on each plate. A clover leaf-type indentation at the intersection of the test organism and Escherichia Coli ATCC 25299 within the zone of inhibition around the carbapenem disc was considered positive for carbapenemase production. K. pneumoniae ATCC BAA-1705 and Escherichia Coli ATCC 25299 was used as the positive and negative control, respectively.

The isolates that were resistant to meropenem, ertapenem, or imipenem were further tested for MBL production using the combined disc method. Carbapenem/ethylenediaminetetraacetic acid (EDTA) $(10 / 930 \mu \mathrm{g})$ combined discs (BIORAD, Munich, Germany) of meropenem + EDTA, ertapenem + EDTA, and imipenem + EDTA were placed $20 \mathrm{~mm}$ from $10 \mu \mathrm{g}$ discs of meropenem, ertapenem, and imipenem, respectively. An increased inhibition zone size $\geq 7 \mathrm{~mm}$ with carbapenem/EDTA was compared to the carbapenem discs alone and was taken as MBL-positive [29].

\subsection{Genotypic Techniques}

Bacterial DNA extraction was carried out using the crude boiling method. Bacterial isolates were subcultured on LB agar (Oxoid, Basingstoke Hampshire, UK) and incubated overnight. Isolate (1-2 colonies) were picked and emulsified in $100 \mu \mathrm{L}$ of sterile distilled water contained in a $1.5 \mathrm{~mL}$ sterile Eppendorf tube. Samples were then boiled for $10 \mathrm{~min}$ at $100^{\circ} \mathrm{C}$ to allow for cell lysis. The lysates were then centrifuged at $4000 \mathrm{rpm}$ for $5 \mathrm{~min}$ at $4{ }^{\circ} \mathrm{C}$. The supernatant was transferred into a new tube, and $3 \mu \mathrm{L}$ of this served as the template for subsequent polymerase chain reaction (PCR) reactions.

Selected ESBL (TEM, SHV, CTX-M) and carbapenemase-encoding genes (OXA-48, NDM, IMP, VIM, and KPC) were detected by PCR for all isolates with primers (Sangong, Shanghai, China) listed in Table 1. PCR was carried out using thermal cycler (BioRad, Hercules, CA, USA) with a total reaction volume of $20.0 \mu \mathrm{L}$ containing 3.0 $\mu \mathrm{L}$ DNA template and $4.0 \mu \mathrm{L}$ of Tag 5X Master Mix (New England Biolabs), $0.5 \mu \mathrm{L} \mathrm{MgCl} 2,0.7 \mu \mathrm{L}$ of $10 \mu \mathrm{mol} \mathrm{L}^{-1}$ forward primer, $0.7 \mu \mathrm{L}$ of $\mu \mathrm{mol} \mathrm{L}^{-1}$ reverse primer, and $11.1 \mu \mathrm{L}$ $\mathrm{ddH}_{2} \mathrm{O}$. Cyclin condition for SHV, CTX-M, KPC, NDM, IMP, VIM, and OXA48 were set at $95^{\circ} \mathrm{C}$ for initial denaturation for $5 \mathrm{~min}$. The second step included 35 cycles of denaturation at $95^{\circ} \mathrm{C}$ for $30 \mathrm{~s}$, annealing at $55^{\circ} \mathrm{C}$ for $45 \mathrm{~s}$, and elongation at $72{ }^{\circ} \mathrm{C}$ for $45 \mathrm{~s}$. This was followed by a final extension temperature of $72{ }^{\circ} \mathrm{C}$ for $7 \mathrm{~min}$. Cyclin conditions for TEM was set at $95^{\circ} \mathrm{C}$ for initial denaturation for $5 \mathrm{~min}$. The second step included 35 cycles of denaturation at $95^{\circ} \mathrm{C}$ for $1 \mathrm{~min}$, annealing at $55^{\circ} \mathrm{C}$ for $1 \mathrm{~min}$, and elongation at $72{ }^{\circ} \mathrm{C}$ for $1 \mathrm{~min}$. ESBL and carbapenemase controls described elsewhere were used [14,30]. Amplified products were detected by electrophoresis using $1 \%$ agarose gel in Tris-acetate-EDTA (TAE) buffer and visualized with ethidium bromide staining (BDH Prolabo, Leuven, Belgium). 
Table 1. Primer sequence used for amplification of extended spectrum $\beta$-lactamase (ESBL) and carbapenemase genes.

\begin{tabular}{|c|c|c|c|}
\hline Name & Primer Sequence $\left(5^{\prime} \rightarrow 3^{\prime}\right)$ & Amplicon Size (bp) & Reference \\
\hline TEM & $\begin{array}{c}\text { Forward-ATGAGTATTCAACATTTCCG } \\
\text { Reverse-TTACCAATGCTTAATCAGTGAG }\end{array}$ & 861 & [31] \\
\hline SHV & $\begin{array}{l}\text { Forward-TCAGCGAAAAACACCTTG } \\
\text { Reverse-TCCCGCAGATAAATCACCA }\end{array}$ & 472 & [31] \\
\hline CTX-M & $\begin{array}{c}\text { Forward-GCGATGGGCAGTACCAGTAA } \\
\text { Reverse-TTACCCAGCGTCAGATTCCG }\end{array}$ & 392 & {$[31]$} \\
\hline KPC & $\begin{array}{c}\text { Forward-CATTCAAGGGCTTTCTTGCTGC } \\
\text { Reverse-ACGACGGCATAGTCATTTGC }\end{array}$ & 538 & {$[32]$} \\
\hline NDM & $\begin{array}{l}\text { Forward-GGTTTGGCGATCTGGTTTTC } \\
\text { Reverse-CGGAATGGCTCATCACGATC }\end{array}$ & 521 & {$[33]$} \\
\hline IMP & $\begin{array}{c}\text { Forward-TTGACACTCCATTTACAG } \\
\text { Reverse-GATTGAGAATTAAGCCACTCT }\end{array}$ & 232 & {$[34]$} \\
\hline VIM & $\begin{array}{c}\text { Forward-TTATGGAGCAACCGATGT } \\
\text { Reverse-CAAAAGTCCCGCTCCAACGA }\end{array}$ & 920 & [35] \\
\hline OXA-48 & $\begin{array}{c}\text { Forward-GCTTGATCGCCCTCGATT } \\
\text { Reverse-GATTTGCTCCGTTGGCCAAA }\end{array}$ & 281 & [36] \\
\hline
\end{tabular}

\subsection{Data Analysis}

Chi-square analysis was used to compare categorical variables. Fisher's exact test was used for cells with expected count below 5. All analyses were done using IBM statistical package for social sciences (SPSS) version 21 (IBM corporation, New York, NY, USA).

\section{Results}

\subsection{Study Population}

The study identified 139 Klebsiella isolates, of which 70/139 (50.4\%), 21/139 (15.1\%), 26/139 $(18.7 \%), 10 / 139(7.1 \%)$, and 12/139 (8.6\%) isolates were obtained from Komfo Anokye Teaching Hospital, Cape Coast Teaching Hospital, Effia Nkwanta Regional Hospital, Tamale Teaching Hospital, and Bolgatanga Regional Hospital, respectively. Of the 139 isolates, 48 (34.5\%) were K. oxytoca, whereas 91 (65.5\%) were K. pneumoniae. The isolates were mainly recovered from blood 18/139 (12.9\%), high vaginal swab (HVS) 12/139 (8.6\%), sputum 20/139 (14.4\%), urine 63/139 (45.3), wound 18/139 (12.9), and "others" [8/139 (5.8\%) ear swabs, pleural aspirates, urethral swab, tracheal aspirates, and cholestetial] (Table 2).

Table 2. Frequency of Klebsiella isolates from different specimen types at the five different hospitals across Ghana.

\begin{tabular}{|c|c|c|c|c|c|c|c|}
\hline \multirow{2}{*}{ Facility } & \multicolumn{6}{|c|}{ Specimen n (\%) } & \multirow{2}{*}{ Total } \\
\hline & Blood & HVS & Sputum & Urine & Wound Swab & Others * & \\
\hline \multicolumn{8}{|l|}{$\begin{array}{l}\text { Northern belt } \\
\text { TTH }\end{array}$} \\
\hline Klebsiella oxytoca & 0 & 0 & $2(66.7)$ & $2(50.0)$ & 0 & 0 & $4(40.0)$ \\
\hline $\begin{array}{c}\text { Klebsiella pneumoniae } \\
\text { BRH }\end{array}$ & $1(100)$ & $1(100)$ & $1(33.3)$ & $2(50.0)$ & 0 & $1(100)$ & $6(60.0)$ \\
\hline Klebsiella oxytoca & 0 & 0 & 0 & $3(30.0)$ & 0 & $1(50.0)$ & $4(33.3)$ \\
\hline $\begin{array}{c}\text { Klebsiella pneumoniae } \\
\text { Middle belt } \\
\text { KATH }\end{array}$ & 0 & 0 & 0 & $7(70.0)$ & 0 & $1(50.0)$ & $8(66.7)$ \\
\hline Klebsiella oxytoca & $3(21.4)$ & 0 & $2(20.0)$ & $13(39.4)$ & $2(20.0)$ & 0 & $20(28.6)$ \\
\hline $\begin{array}{l}\text { Klebsiella pneumoniae } \\
\text { Southern belt }\end{array}$ & $11(78.6)$ & 0 & $8(80.0)$ & $20(60.6)$ & $8(80.0)$ & $3(100)$ & $50(71.4)$ \\
\hline
\end{tabular}


Table 2. Cont.

\begin{tabular}{|c|c|c|c|c|c|c|c|}
\hline \multirow{2}{*}{ Facility } & \multicolumn{6}{|c|}{ Specimen n (\%) } & \multirow{2}{*}{ Total } \\
\hline & Blood & HVS & Sputum & Urine & Wound Swab & Others * & \\
\hline ССТH & & & & & & & \\
\hline Klebsiella oxytoca & $1(33.3)$ & $3(100)$ & 0 & $3(37.5)$ & $3(50.0)$ & 0 & $10(47.6)$ \\
\hline $\begin{array}{c}\text { Klebsiella pneumoniae } \\
\text { EFNTH }\end{array}$ & $2(66.7)$ & 0 & 0 & $5(62.5)$ & $3(50.0)$ & $1(100)$ & $11(52.4)$ \\
\hline Klebsiella oxytoca & 0 & $2(25.0)$ & $3(42.9)$ & $3(37.5)$ & $2(100)$ & 0 & $10(38.5)$ \\
\hline Klebsiella pneumoniae & 0 & $6(75.0)$ & $4(57.1)$ & $5(62.5)$ & 0 & $1(100)$ & $16(61.5)$ \\
\hline Total & $18(100)$ & $12(100)$ & $20(100)$ & $63(100)$ & $18(100)$ & $8(100)$ & $139(100)$ \\
\hline
\end{tabular}

KATH = Komfo Anokye Teaching Hospital, CCTH = Cape Coast Teaching Hospital, EFNTH = Effia Nkwanta Teaching Hospital, TTH = Tamale Teaching Hospital, BRH = Bolgatanga Regional Hospital. Others * = ear swabs, pleural aspirates, urethral swab, tracheal aspirates, and cholestetial fluid.

\subsection{Antimicrobial Susceptibility Profile of Isolates}

The isolates were tested against a panel of 16 antibiotics comprising $10 \beta$-lactam- and six non- $\beta$-lactam-based drugs. High resistance to ampicillin was observed for K. oxytoca $47 / 48(97.9 \%)$ and K. pneumoniae $91 / 91$ (100\%). There was also a greater than $70 \%$ resistance against the 3rd-generation cephalosporins (ceftriaxone, cefotaxime, and ceftazidime) by both species. The carbapenem-based drugs were relatively active compared to the other classes of $\beta$-lactam drugs. Among the carbapenem-based drugs, meropenem was the least active agent, recording 18/48 (37.5\%) and 27/91 (29.7\%) resistance against K. oxytoca and K. pneumoniae, respectively. The most active carbapenem agent was imipenem, producing 5/48 (10.4\%) and 13/91 (14.3\%) resistance against K. oxytoca and K. pneumoniae, respectively. The most active non- $\beta$-lactam was amikacin. Except for amikacin, to which just $1 / 48$ (2.1\%) K. oxytoca and $4 / 91(4.4 \%)$ K. pneumoniae showed resistance, high resistance to the other non- $\beta$-lactam antibiotics was recorded for K. oxytoca (54.2\%-89.6\%) and K. pneumoniae (73.6\%-91.2\%) (Table 3).

Table 3. Antimicrobial susceptibility profile of Klebsiella oxytoca and Klebsiella pneumoniae.

\begin{tabular}{|c|c|c|c|c|c|c|c|c|}
\hline \multirow{2}{*}{ Variable } & \multicolumn{3}{|c|}{ K. oxytoca $(n=48)$} & \multirow{2}{*}{$p$-Value } & \multicolumn{3}{|c|}{ K. pneumoniae $(n=91)$} & \multirow{2}{*}{$p$-Value } \\
\hline & R n (\%) & I n (\%) & S n (\%) & & R n (\%) & I n (\%) & S n (\%) & \\
\hline \multicolumn{9}{|c|}{$\begin{array}{l}\beta \text {-lactams } \\
\text { Penicillin }\end{array}$} \\
\hline Ampicillin & $47(97.9)$ & $1(2.1)$ & $0(0.0)$ & $<0.001$ & $91(100)$ & $0(0.0)$ & $0(0.0)$ & $<0.001$ \\
\hline \multicolumn{9}{|c|}{ 3rd-gen cephalosporin } \\
\hline Ceftriaxone & $40(83.3)$ & $3(6.3)$ & $5(10.4)$ & $<0.001$ & 83 (91.2) & $7(7.7)$ & $1(1.1)$ & $<0.001$ \\
\hline Cefotaxime & $44(91.7)$ & $2(4.2)$ & $2(4.2)$ & $<0.001$ & $70(76.9)$ & $8(8.8)$ & $13(14.3)$ & $<0.001$ \\
\hline Ceftazidime & $38(79.2)$ & $4(8.3)$ & $6(12.5)$ & $<0.001$ & $65(71.4)$ & $15(16.5)$ & $11(12.1)$ & $<0.001$ \\
\hline \multicolumn{9}{|c|}{ 2nd-gen cephalosporin } \\
\hline Cefuroxime & $42(87.5)$ & $3(6.3)$ & $3(6.3)$ & $<0.001$ & $90(98.9)$ & $1(2.1)$ & $0(0.0)$ & $<0.001$ \\
\hline Cefoxitin & 18 (37.5) & $11(22.9)$ & 19 (39.5) & 0.168 & 31 (36.5) & $24(28.2)$ & 30 (35.3) & 0.480 \\
\hline Cefotetan & $14(29.2)$ & $1(2.1)$ & $33(68.8)$ & $<0.001$ & $18(19.8)$ & $4(4.4)$ & $69(75.8)$ & $<0.001$ \\
\hline \multicolumn{9}{|c|}{ Carbapenem } \\
\hline Meropenem & $18(37.5)$ & $9(18.8)$ & $21(43.8)$ & 0.026 & $27(29.7)$ & $25(27.5)$ & $39(42.9)$ & 0.059 \\
\hline Imipenem & 5 (10.4) & $0(0.0)$ & 43 (89.6) & $<0.001$ & 13 (14.3) & $1(1.1)$ & 77 (84.6) & $<0.001$ \\
\hline Ertapenem & $7(14.6)$ & $9(18.8)$ & $32(66.7)$ & $<0.001$ & $15(16.5)$ & $14(15.4)$ & $62(68.1)$ & $<0.001$ \\
\hline \multicolumn{9}{|c|}{ Non- $\beta$-lactams } \\
\hline Tetracycline & $41(85.4)$ & $3(6.3)$ & $4(8.3)$ & $<0.001$ & 77 (84.6) & $1(1.1)$ & $13(14.3)$ & $<0.001$ \\
\hline Cotrimoxazole & 43 (89.6) & - & $5(10.4)$ & $<0.001$ & $83(91.2)$ & $0(0.0)$ & $8(8.8)$ & $<0.001$ \\
\hline Ciprofloxacin & $33(68.8)$ & 15 (31.3) & $0(0.0)$ & $<0.001$ & $69(75.8)$ & $22(24.2)$ & $0(0.0)$ & $<0.001$ \\
\hline Chloramphenicol & $26(54.2)$ & $4(8.3)$ & $18(37.5)$ & $<0.001$ & $67(73.6)$ & $4(4.4)$ & $20(22)$ & $<0.001$ \\
\hline Gentamicin & $31(64.6)$ & $4(8.3)$ & $13(27.1)$ & $<0.001$ & $67(73.6)$ & $3(3.3)$ & $21(23.1)$ & $<0.001$ \\
\hline Amikacin & $1(2.1)$ & $0(0.0)$ & 47 (97.9) & $<0.001$ & $4(4.4)$ & $0(0.0)$ & 87 (95.6) & $<0.001$ \\
\hline
\end{tabular}


3.3. Phenotypic Distribution of ESBL-, AmpC-, MBL-, and Carbapenemase-Producing Klebsiella Isolate by Specimen Type

There was no evidence of any association $(p>0.005)$ between ESBL, AmpC, and MBL production and the species of Klebsiella (Table 4). On the other hand, the proportion of carbapenemase producers within K. oxytoca species 6/48 $(12.5 \%)$ was significantly $(p<0.05)$ lower compared to the proportion of carbapenemase producers within K. pneumoniae species 24/91 (26.4\%). ESBL producers were most abundant in "others" [6/8 (75.0\%), ear swabs, pleural aspirates, urethral swab, tracheal aspirates, and cholestetial fluid] and least abundant in HVS 7/12 (58.3\%). HVS 5/12 (41.7\%) represented the specimen type that harbored the most AmpC producers, whereas sputum, wound swab, and "others" recorded no $(0 \%)$ AmpC producers. MBL producers were predominantly recovered from HVS $4 / 12(33.3 \%)$. However, wound swab 5/18 (27.8\%) served as the source that harboured the most carbapenemase producers. Details of the distribution of $\beta$-lactamase producers of $K$. oxytoca and $K$. pneumoniae are presented in Table 4.

\subsection{Phenotypic Distribution of ESBL-, AmpC-, and Carbapenemase-Producing Klebsiella Species by Geographical Location}

A geographical decreasing gradient of ESBL and carbapenemase production was observed from the northern belt to the southern belt as opposed to an increasing gradient of MBL production from the northern to the southern belt. The production of AmpC was highest in the northern belt, followed by the southern and middle belts.

The overall carbapenemase producers were found to be 30/139 (21.5\%). The highest resistance mechanism among the Klebsiella isolate was ESBL 99/139 (71.2\%), followed by MBL 23/139 (16.5\%), and AmpC 27/139 (19.4\%). The ESBL resistance observed at health facilities in the northern belt significantly differed from that of the middle belt $(p=0.012)$ and southern belt $(p=0.001)$ but not between the middle and southern belts. There were no significant regional differences in the production of AmpC, carbapenemase, and MBL $(p>0.05)$. Figure 1 gives a summary of the geographical distribution of ESBL, AmpC, carbapenemase, and MBL production among Klebsiella isolates from health facilities in Ghana.

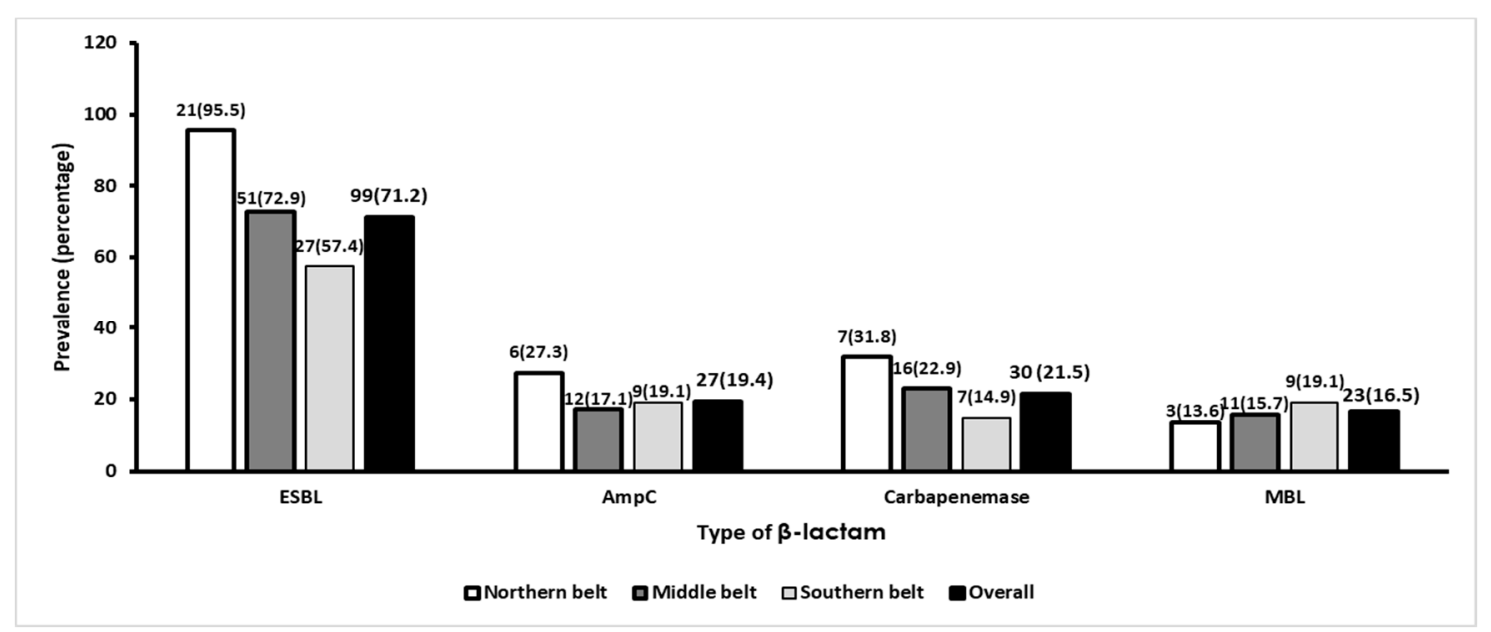

Figure 1. Phenotypic distribution of ESBL-, AmpC-, carbapenemase-, and MBL-producing Klebsiella isolates from referral health facilities in Ghana. 
Table 4. Prevalence of ESBL-, AmpC $\beta$-lactamase (AmpC)-, metallo- $\beta$-lactamase (MBL)- and carbapenemase-producing Klebsiella species by specimen, Ghana.

\begin{tabular}{|c|c|c|c|c|c|c|c|c|c|}
\hline Resistance Mechanism & Species & $\begin{array}{l}\text { Within } \\
\text { Species }\end{array}$ & Total & $\begin{array}{l}\text { Blood } \\
n=18\end{array}$ & $\begin{array}{l}\text { HVS } \\
n=12\end{array}$ & $\begin{array}{c}\text { Sputum } \\
n=20\end{array}$ & $\begin{array}{l}\text { Urine } \\
n=63\end{array}$ & $\begin{array}{c}\text { Wound Swab } \\
n=18\end{array}$ & $\begin{array}{c}\text { Others } \\
n=8\end{array}$ \\
\hline ESBL producers & K.O & $35 / 48(72.9)^{\#}$ & $35 / 99(35.4)$ & $4 / 4(100)$ & 2/5 (40.0) & 5/7 (71.4) & 17/24 (70.8) & 6/7 (85.7) & 1/1 (100) \\
\hline$n=99$ & K.P & 64/91 (70.3) & $64 / 99(64.6)$ & 9/14 (64.2) & $5 / 7(71.4)$ & $9 / 13(69.2)$ & $29 / 39(74.3)$ & $7 / 11(63.6)$ & $5 / 7(71.4)$ \\
\hline Overall prevalence (\%) & & $99 / 139$ (71.2) & $(100)$ & $13 / 18(72.2)$ & $7 / 12(58.3)$ & $14 / 20(70.0)$ & $46 / 63(73.0)$ & $13 / 18(72.2)$ & $6 / 8(75.0)$ \\
\hline AmpC producers & K.O & $8 / 48(16.6)^{\#}$ & $8 / 27(29.6)$ & $3 / 4(75.0)$ & $2 / 5(40.0)$ & $0 / 7(0)$ & $3 / 24(12.5)$ & $0 / 7(0)$ & $0 / 1(0)$ \\
\hline$n=27$ & K.P & $19 / 91(20.8)$ & 19/27 (70.4) & $1 / 14(7.1)$ & $3 / 7(42.9)$ & $5 / 13(38.5)$ & 7/39 (17.9) & 2/11 (18.1) & $1 / 7(14.3)$ \\
\hline Overall prevalence (\%) & & $27 / 139(19.4)$ & (100) & $4 / 18(22.2)$ & $5 / 12(41.7)$ & $5 / 20(25.0)$ & $10 / 63(15.9)$ & 2/18 (11.1) & $1 / 8(12.5)$ \\
\hline MBL producers & K.O & $5 / 48(10.4)^{\#}$ & $5 / 23(21.7)$ & $0 / 4$ & $1 / 5(20.0)$ & 2/7 (28.6) & $1 / 24(4.1)$ & $1 / 7(14.3)$ & $0 / 1$ \\
\hline$n=23$ & K.P & $18 / 91(19.8)$ & $18 / 23(78.3)$ & $3 / 14(21.1)$ & $3 / 7(42.9)$ & $3 / 13(23.1)$ & $3 / 39(7.7)$ & $4 / 11(36.4)$ & $2 / 7(28.6)$ \\
\hline Overall prevalence (\%) & & 23/139 (16.5) & $(100)$ & 3/18 (16.7) & 4/12 (33.3) & $5 / 20(25.0)$ & $4 / 63(6.3)$ & $5 / 18(27.8)$ & $2 / 8(25.0)$ \\
\hline Carbapenemase producers & K.O & $6 / 48(12.5)^{*}$ & $6 / 30(20.0)$ & $0 / 4(0)$ & $1 / 5(20.0)$ & $1 / 7(14.3)$ & $4 / 24(16.7)$ & 0/7 (0) & $0 / 1(0)$ \\
\hline$n=30$ & K.P & $24 / 91(26.4)$ & $24 / 30(80.0)$ & $4 / 14(28.6)$ & $3 / 7(42.9)$ & $4 / 13(30.8)$ & $12 / 39(30.8)$ & $5 / 11(45.5)$ & $2 / 7(28.6)$ \\
\hline Overall prevalence (\%) & & $30 / 139(21.6)$ & (100) & $4 / 18(22.2)$ & $4 / 12(33.3)$ & $5 / 20(25.0)$ & $16(25.4)$ & $5 / 18(27.8)$ & $2 / 8(25.0)$ \\
\hline
\end{tabular}

$\mathrm{K} . \mathrm{O}=$ Klebsiella oxytoca, $\mathrm{K} . \mathrm{P}=$ Klebsiella pneumoniae, Total = producers in a particular species expressed as a percentage $(\%)$ of the total number of producers in the study, HVS = high vagina swab. Chi-square analysis was used to compare all categorical variables at an alpha value of 0.05 . Fisher's exact test was used for cells with counts less than 5 . Data is presented as count $(\%)^{\#}=$ no significant difference comparing producers of K.O and K.P., ${ }^{*}=$ significant difference comparing producers of K.O and K.P. Others $=$ ear swabs, pleural aspirates, urethral swab, tracheal aspirates, and cholestetial fluid. 


\subsection{Genotypic Distribution of bla ESBL-Encoding Genes in Klebsiella Species}

There was no evidence of association $(p>0.05)$ between the occurrence of bla ESBL genes (TEM, SHV, CTX-M) and the Klebsiella species. However, the proportion of TEM-positive Klebsiella isolates from the facilities within the northern belt 9/22 (40.9) was significantly higher $(p=0.032)$ than the proportion of TEM-positive Klebsiella isolate from health facilities within the southern belt 8/47 $(17.0 \%)$. Furthermore, the proportion of SHV-positive isolates in the health facility at the middle belt $25 / 70(35.7 \%)$ was significantly higher $(p=0.053)$ than the proportion of SHV-positive isolates from health facilities within the southern belt 9/47 (19.1\%). bla TEM, bla SHV, and bla CTX-M were most abundant in sputum $7 / 20(35.0 \%)$, HVS 4/12 (33.3\%) and blood 6/18 (33.3\%), respectively. Details of the distribution of bla ESBL genes by Klebsiella species, geographical location of health facility, and specimen are presented in Table 5.

Table 5. Distribution of bla ESBL genes by Klebsiella species and geographical location.

\begin{tabular}{|c|c|c|c|}
\hline \multirow{2}{*}{ Variable } & \multicolumn{3}{|c|}{ BlaESBL } \\
\hline & TEM $(n=36)$ & SHV $(n=40)$ & CTX-M $(n=37)$ \\
\hline \multicolumn{4}{|l|}{ Species } \\
\hline Klebsiella oxytoca $(n=48)$ & $11 / 48(22.9 \%)$ & $13 / 48(27.1 \%)$ & $10 / 48(20.8 \%)$ \\
\hline Klebsiella pneumoniae $(n=91)$ & $25 / 91(27.5 \%)$ & $27 / 91(29.7 \%)$ & $27 / 91(29.7 \%)$ \\
\hline$p$-Value ${ }^{\mathrm{a}}$ & 0.560 & 0.749 & 0.262 \\
\hline \multicolumn{4}{|l|}{ Region } \\
\hline Northern belt $(n=22)$ & $9 / 22(40.9 \%)$ & $6 / 22(27.3 \%)$ & $7 / 22(31.8 \%)$ \\
\hline Middle belt $(n=70)$ & $19 / 70(27.1 \%)$ & $25 / 70(35.7 \%)$ & $22 / 70(31.4 \%)$ \\
\hline Southern belt $(n=47)$ & $8 / 47(17.0 \%)$ & $9 / 47(19.1 \%)$ & $8 / 47(17.0 \%)$ \\
\hline$p$-Value $\mathrm{e}^{\mathrm{b}}$ & 0.221 & 0.465 & 0.973 \\
\hline$p$-Value ${ }^{\mathrm{c}}$ & 0.032 & 0.446 & 0.165 \\
\hline $\bar{p}$-Value ${ }^{\mathrm{d}}$ & 0.203 & 0.053 & 0.080 \\
\hline \multicolumn{4}{|l|}{ Specimen } \\
\hline Blood $(n=18)$ & $3 / 18(16.7 \%)$ & $5 / 18(27.8 \%)$ & $6 / 18(33.3 \%)$ \\
\hline $\operatorname{HVS}(n=12)$ & $2 / 12(16.7 \%)$ & $4 / 12(33.3 \%)$ & $3 / 12(25.0 \%)$ \\
\hline Others $(n=8)$ & $1 / 8(12.5 \%)$ & $2 / 8(25.0 \%)$ & $2 / 8(25.0 \%)$ \\
\hline Sputum $(n=20)$ & $7 / 20(35.0 \%)$ & $6 / 20(30.0 \%)$ & $3 / 20(15.0 \%)$ \\
\hline Urine $(n=63)$ & $18 / 63(28.5 \%)$ & $18 / 63(28.5 \%)$ & $19 / 63(30.1 \%)$ \\
\hline Wound swab $(n=18)$ & $5 / 18(27.7 \%)$ & $5 / 18(27.7 \%)$ & $4 / 18(22.2 \%)$ \\
\hline Total & $36 / 139(25.9 \%)$ & $40 / 139(28.8 \%)$ & $37(26.6 \%)$ \\
\hline
\end{tabular}

Chi-square analysis was used to compare all categorical variables at an alpha value of 0.05 . Fisher's exact test was used for cells with counts less than 5. $p$-Value ${ }^{\mathrm{a}}=p$-value for comparing Klebsiella oxytoca with Klebsiella pneumoniae, $p$-value ${ }^{\mathrm{b}}=p$-value for comparing northern belt with middle belt, $p$-value $\mathrm{c}^{\mathrm{c}}=p$-value for comparing northern belt with southern belt, $p$-value ${ }^{\mathrm{d}}=p$-value for comparing middle belt with southern belt.

\subsection{Distribution of Blacarbapenemase-Positive Isolates}

A total of 3/139 (2.1\%) isolates were identified as positive for carbapenemase genes. Of the three isolates, one was positive for harboring two carbapenemase genes, i.e., OXA-48- and NDM-encoding genes, whereas the other two were positive for only OXA-48-encoding genes. No isolate was positive for IMP, VIM, and KPC. Two of the positive isolates were recovered from facilities situated in the southern belt, whereas the remaining one was isolated in the middle belt. Table 6 shows the details of isolates positive for carbapenemase-encoding genes and their respective antibiogram. 
Table 6. Antibiogram of bla carbapenemase-positive Klebsiella isolates.

\begin{tabular}{|c|c|c|c|}
\hline \multirow{2}{*}{$\begin{array}{c}\text { Variable } \\
\text { Carbapenemase }\end{array}$} & \multicolumn{3}{|c|}{ Resistance Profile } \\
\hline & OXA-48, NDM & OXA-48 & OXA-48 \\
\hline ID & EF 41 & EF 141 & КАTH 05 \\
\hline Presence of ESBL & TEM, CTX-M & SHV & - \\
\hline Species & K. pneumoniae & K. pneumoniae & K. pneumoniae \\
\hline Age(years) & 73 & 40 & 57 \\
\hline Specimen & Urine & Sputum & Sputum \\
\hline Hospital & $\begin{array}{c}\text { Effia Nkwanta Regional } \\
\text { Hospital }\end{array}$ & $\begin{array}{c}\text { Effia Nkwanta Regional } \\
\text { Hospital }\end{array}$ & $\begin{array}{c}\text { Komfo Anokye Teaching } \\
\text { Hospital }\end{array}$ \\
\hline $\begin{array}{c}\text { Geographical location } \\
\beta \text {-Lactams }\end{array}$ & Southern belt & Southern belt & Middle belt \\
\hline \multicolumn{4}{|c|}{ 3rd-gen cephalosporin } \\
\hline Ceftriaxone & $\mathrm{R}$ & $\mathrm{R}$ & $S$ \\
\hline Cefotaxime & $\mathrm{R}$ & $\mathrm{R}$ & $S$ \\
\hline Ceftazidime & $\mathrm{R}$ & $\mathrm{R}$ & $\mathrm{R}$ \\
\hline \multicolumn{4}{|c|}{ 2nd-gen cephalosporin } \\
\hline Cefuroxime & $\mathrm{R}$ & $\mathrm{R}$ & $\mathrm{R}$ \\
\hline Cefoxitin & $S$ & I & $S$ \\
\hline Cefotetan & $S$ & $S$ & $\mathrm{R}$ \\
\hline \multicolumn{4}{|l|}{ Carbapenems } \\
\hline Meropenem & I & $\mathrm{R}$ & $\mathrm{R}$ \\
\hline Imipenem & $\mathrm{R}$ & $\mathrm{R}$ & $\mathrm{R}$ \\
\hline Ertapenem & $S$ & S & $\mathrm{R}$ \\
\hline \multicolumn{4}{|l|}{ Non- $\beta$-lactams } \\
\hline Ampicillin & $\mathrm{R}$ & $\mathrm{R}$ & $\mathrm{R}$ \\
\hline Tetracycline & S & $\mathrm{R}$ & $\mathrm{R}$ \\
\hline Cotrimoxazole & $\mathrm{R}$ & $\mathrm{R}$ & $S$ \\
\hline Ciprofloxacin & $\mathrm{R}$ & $\mathrm{R}$ & $\mathrm{R}$ \\
\hline Chloramphenicol & $\mathrm{R}$ & $\mathrm{R}$ & S \\
\hline Gentamicin & $\mathrm{R}$ & $\mathrm{R}$ & $\mathrm{R}$ \\
\hline Amikacin & S & $S$ & $S$ \\
\hline
\end{tabular}

\subsection{Agreement between Phenotypic Tests and Genotypic Test}

Of the 72 genotypically positive ESBL isolates, only 51 (70.8\%) were phenotypically positive. Of the 21 apparent false-negative ESBLs, 12 were phenotypically positive for AmpC production. All three genotypically carbapenemase-positive isolates were negative for MHT. However, two (EF 41 and EF I41) were positive for MBL production.

\section{Discussion}

Klebsiella spp. are an important cause of multidrug-resistant infections with varying reports of $\beta$-lactam antimicrobial resistance levels worldwide. This study described the distribution of ESBL, AmpC, carbapenemase, and MBL resistance mechanisms by geographical location of the health facilities in Ghana.

The most recent nationwide antimicrobial resistance survey in Ghana involving a broad spectrum of bacteria isolates showed that isolates from healthcare facilities in the northern belt had generally higher resistance profile compared to the middle and southern belts [37]. The investigators of the survey found a statistically significant $(p<0.05)$ difference in antimicrobial resistance levels between isolates from the northern and southern belts. Likewise, the present study documented the highest prevalence of ESBL, AmpC, and carbapenemase production in Klebsiella isolates from health facilities situated within the northern belt. Similar to the nationwide survey, the present study reported a significantly $(p<0.05)$ higher ESBL production in the northern belt compared to not only the southern 
belt but also the middle belt. This may be a reflection of the relatively higher genotypic levels of TEM and CTX-M in the northern belt, as shown by this study.

The prevalence of ESBL, carbapenemase, and MBL producers recorded in the healthcare facility in the middle belt was in between that recorded in the northern and southern belts. This could reflect the arbitrary moderate temperatures and socioeconomic burden in the middle belt of Ghana. Notably, isolates positive for carbapenemase-encoding genes were detected in facilities situated in the middle belt (one isolate) and southern belt (two isolates) but none in the northern belt. This contradicts the phenotypic finding that indicated a higher prevalence of carbapenemase production in the northern belt. Perhaps the presence of other resistance mechanism or carbapenemase-resistant genes not explored by this study could explain these two paradoxical findings. The factors underlining the observed varying levels of $\beta$-lactam resistance mechanisms could be accounted for by the difference in temperatures and socioeconomic status in these regions. It is postulated that high levels of resistant pathogens correlate with regions with warmer temperatures [23,24]. In the case of K. pneumoniae, it is estimated that a $10{ }^{\circ} \mathrm{C}$ change in temperature is associated with a $2.2 \%$ increase in resistance [23]. One model aimed at assessing the vulnerability of Ghana with respect to climate change estimated a temperature increase of 1.5 to $3.0^{\circ} \mathrm{C}$ by 2060 , with the northern belt expected to be the most affected zone [38]. In Ghana, the northern belt is the warmest belt [22], and this could contribute to the high resistance observed in the north. In addition, antimicrobial resistance-driving factors, including self-medication, prescription of substandard antimicrobials, and poor sanitation and hygiene have been shown to be associated with low socioeconomic status $[39,40]$. The northern belt of Ghana is deemed the poorest of the three belts in terms of economic status [21], and this could partly contribute to the relatively high resistance observed in this region.

A recent study documented the presence of carbapenem resistance in $2.9 \%$ of Gram-negative bacteria [41]. However, in the present study, not all the carbapenem-resistant isolates tested positive with MHT, suggesting that more than 30 isolates $(>21.5 \%)$ are resistant to one or more carbapenem drugs. The reports from these two studies are worrisome because, in Ghana, carbapenems are usually reserved for the treatment of serious infections, and as such resistance to carbapenems is expected to be low. The difference between these two reports could be explained by the fact that the prevalence estimated by Codjoe et al. was among a broad-spectrum Gram-negative isolates, whereas the one estimated by the present study was the prevalence among Klebsiella isolates alone. Also, the difference in sample size might have partly contributed to the discrepancies between these two studies. Codjoe and his colleagues identified two OXA-48-encoding genes in K. pneumoniae and NDM-encoding genes in Pseudomonas aeruginosa and Acinetobacter spp. [41]. In concordance with this earlier study [41], three OXA-48 carbapenemase-positive Klebsiella isolates were identified by the present study, of which one was also positive for NDM-encoding gene. Findings from this study, together with the findings of Codjoe et al. [41], might suggest a possible ongoing interspecies dissemination of NDM in Ghana. K. pneumoniae coharboring NDM and OXA-48 have also been previously documented in Tunisia and Uganda [14,42]. This indicates how common K. pneumoniae isolates coharboring NDM and OXA-48 are reported in Africa.

The study recorded 21/72 false-negative ESBL production, of which 12 were phenotypically positive for AmpC production. This could be due to the concomitant expression of ESBL and plasmid-mediated AmpC [43]. In addition, of the 99 phenotypically positive ESBL isolates, only 51 were genotypically positive for ESBL-encoding genes. This suggests a possible resistance by non-ESBL genes or the presence of other possible atypical ESBL genes, which were not explored by the study. All three genotypically positive carbapenemase producers were negative with MHT, reiterating the poor performance of MHT. However, isolate EF-41 harboring NDM and OXA-48 was identified as positive with MBL test. In addition, EF-141 (positive for only OXA-48 carbapenemase) was also positive for MBL test, suggesting the presence of other possible MBL genes not explored by the present study. 
The findings of this study remain observational as factors including the limited number of isolates used, the restricted choice of bacteria isolates, and the short period within which the study was carried out might have potentially affected the outcome. For instance, the sample size in the present study, especially in the northern belt, might not be adequately powered to estimate the geographical differences in certain $\beta$-lactamases, including carbapenemase and AmpC. Another limitation is that the presence of specific AmpC-resistant genes was not tested by the present study.

\section{Conclusions}

There were variations in $\beta$-lactam resistance among Klebsiella spp. from health facilities situated in the northern, middle, and southern belts of Ghana. $\beta$-Lactam resistance at the health facilities situated within the northern belt was considerably higher compared to health facilities located within the middle and southern belts. The detection of OXA-48- and NDM-resistant genes could signal the commencement of carbapenemase dissemination, adding to the already alarmingly high prevalence of ESBL-specific genes in Ghana. This study provides preliminary evidence that emphasizes the need to direct more attention to antimicrobial resistance monitoring and surveillance, especially in the northern belt of Ghana. This would be critical for creating and fine-tuning effective antimicrobial resistance control strategies and for informing accurate antibiotic prescription in clinical settings.

\section{Future Perspective}

This study serves as a baseline study for further studies targeting different bacteria isolates and different antimicrobial resistance mechanisms. Although the study provides insight into the phenotypic and genotypic prevalence of ESBL, AmpC, and carbapenemase and their regional distribution in Klebsiella isolates, little work has been done to molecularly characterize carbapenemase genes in Ghana. Future perspective may include molecular characterization of NDM- and OXA-48-producing K. pneumoniae in Ghana. In addition, molecular testing could be performed to characterize the resistance profile to amikacin against Klebsiella isolates.

Author Contributions: E.Q., O.C., and E.A. carried out the laboratory work. P.A.B., D.O.A., and I.D. won a grant for the work. P.A.B., D.O.A., D.O.-Y., and I.D. supervised all aspects of the laboratory work. R.O.M. provided all the controls for the work and also provided guidance in the laboratory. E.Q. performed all statistical analyses. E.Q. prepared the manuscript. I.D. and P.A.B. reviewed the manuscript. All authors read and approved the manuscript.

Funding: This work was funded by the Directorate of Research Innovation and Consultancy (DRIC) of the University of Cape Coast.

Acknowledgments: We would like to thank the heads and laboratory staff of the health facilities we used. We also thank the Department of Biomedical Sciences for the use of their laboratory.

Conflicts of Interest: The authors declare that they have no conflict of interest.

\section{References}

1. Pitout, J.D.D.; Nordmann, P.; Poirel, L. Carbapenemase-Producing Klebsiella pneumoniae, a Key Pathogen Set for Global Nosocomial Dominance. Antimicrob. Agents Chemother. 2015, 59, 5873-5884. [CrossRef] [PubMed]

2. Singh, L.; Cariappa, M.; Kaur, M. Klebsiella oxytoca: An emerging pathogen? Med. J. Armed Forces India 2016, 72, S59-S61. [CrossRef] [PubMed]

3. Agyepong, N.; Govinden, U.; Owusu-Ofori, A.; Essack, S.Y. Multidrug-resistant gram-negative bacterial infections in a teaching hospital in Ghana. Antimicrob. Resist. Infect. Control. 2018, 7, 37. [CrossRef] [PubMed]

4. Boye, A.; Siakwa, P.M.; Boampong, J.N.; Koffuor, G.A.; Ephraim, R.K.D.; Amoateng, P.; Penu, D. Asymptomatic urinary tract infections in pregnant women attending antenatal clinic in Cape Coast, Ghana. E3 J. Med. Res. 2012, 1, 74-83.

5. Acquah, S.E.; Quaye, L.; Sagoe, K.; Ziem, J.B.; Bromberger, P.; Amponsem, A. Susceptibility of bacterial etiological agents to commonly-used antimicrobial agents in children with sepsis at the Tamale Teaching Hospital. BMC Infect. Dis. 2013, 13, 89. [CrossRef] [PubMed] 
6. Codjoe, F.S.; Donkor, E.S. Carbapenem Resistance: A Review. Med. Sci. 2018, 6, 1. [CrossRef] [PubMed]

7. Thomson, K.S. Controversies about extended-spectrum and AmpC beta-lactamases. Emerg. Infect. Dis. 2001, 7, 333-336. [CrossRef] [PubMed]

8. Tzouvelekis, L.S.; Markogiannakis, A.; Psichogiou, M.; Tassios, P.T.; Daikos, G.L. Carbapenemases in Klebsiella pneumoniae and Other Enterobacteriaceae: An Evolving Crisis of Global Dimensions. Clin. Microbiol. Rev. 2012, 25, 682-707. [CrossRef] [PubMed]

9. Livermore, D.M. Bacterial Resistance: Origins, Epidemiology, and Impact. Clin. Infect. Dis. 2003, 36, 11-23. [CrossRef] [PubMed]

10. Chaudhary, U.; Aggarwal, R. Extended spectrum B-lactamases (ESBL)-An emerging threat to clinical therapeutics. Indian J. Med. Microbiol. 2004, 22, 75-80.

11. Notake, S.; Matsuda, M.; Tamai, K.; Yanagisawa, H.; Hiramatsu, K.; Kikuchi, K. Detection of IMP Metallo- $\beta$-Lactamase in Carbapenem-Nonsusceptible Enterobacteriaceae and Non-Glucose-Fermenting Gram-Negative Rods by Immunochromatography Assay. J. Clin. Microbiol. 2013, 51, 1762-1768. [CrossRef] [PubMed]

12. Sangare, S.A.; Rondinaud, E.; Maataoui, N.; Maiga, A.I.; Guindo, I.; Camara, N.; Dicko, O.A.; Dao, S.; Diallo, S.; Bougoudogo, F.; et al. Very high prevalence of extended-spectrum beta-lactamase-producing Enterobacteriaceae in bacteriemic patients hospitalized in teaching hospitals in Bamako, Mali. PLoS ONE 2017, 12, e0172652. [CrossRef] [PubMed]

13. Ouedraogo, A.-S.; Sanou, M.; Kissou, A.; Sanou, S.; Solaré, H.; Kaboré, F.; Poda, A.; Aberkane, S.; Bouzinbi, N.; Sano, I.; et al. High prevalence of extended-spectrum B-lactamase producing enterobacteriaceae among clinical isolates in Burkina Faso. BMC Infect. Dis. 2016, 16, 2. [CrossRef] [PubMed]

14. Okoche, D.; Asiimwe, B.B.; Katabazi, F.A.; Kato, L.; Najjuka, C.F. Prevalence and Characterization of Carbapenem-Resistant Enterobacteriaceae Isolated from Mulago National Referral Hospital, Uganda. PLoS ONE 2015, 10, e0135745. [CrossRef] [PubMed]

15. Poirel, L.; Revathi, G.; Bernabeu, S.; Nordmann, P. Detection of NDM-1-producing Klebsiella pneumoniae in Kenya. Antimicrob. Agents Chemother. 2011, 55, 934-936. [CrossRef] [PubMed]

16. Brink, A.J.; Coetzee, J.; Clay, C.G.; Sithole, S.; Richards, G.A.; Poirel, L.; Nordmann, P. Emergence of New Delhi Metallo-Beta-Lactamase (NDM-1) and Klebsiella pneumoniae Carbapenemase (KPC-2) in South Africa. J. Clin. Microbiol. 2012, 50, 525. [CrossRef] [PubMed]

17. Feglo, P.; Adu-Sarkodie, Y. Antimicrobial Resistance Patterns of Extended Spectrum B-Lactamase Producing Klebsiellae and E. coli Isolates from a Tertiary Hospital in Ghana. Eur. Sci. J. ESJ 2016, 12, 12.

18. Feglo, P.; Adu-Sarkodie, Y.; Ayisi, L.; Jain, R.; Spurbeck, R.R.; Springman, A.C.; Walk, S.T. Emergence of a Novel Extended-Spectrum- $\beta$-Lactamase (ESBL)-Producing, Fluoroquinolone-Resistant Clone of Extraintestinal Pathogenic Escherichia Coli in Kumasi, Ghana. J. Clin. Microbiol. 2013, 51, 728-730. [CrossRef] [PubMed]

19. Opoku, S.; Feglo, P. AmpC beta-lactamase production among Pseudomonas aeruginosa and Proteus mirabilis isolates at the Komfo Anokye Teaching Hospital, Kumasi, Ghana. J. Microbiol. Antimicrob. 2014, 6, 13-20.

20. Eibach, D.; Campos, C.B.; Krumkamp, R.; Al-Emran, H.M.; Dekker, D.; Boahen, K.G.; Kreuels, B.; Adu-Sarkodie, Y.; Aepfelbacher, M.; Park, S.E.; et al. Extended spectrum beta-lactamase producing Enterobacteriaceae causing bloodstream infections in rural Ghana, 2007-2012. Int. J. Med Microbiol. 2016, 306, 249-254. [CrossRef]

21. United Nations. Ghana Country Analysis. 2010. Available online: https://www.undp.org/content/dam/ghana/ docs/UNDAF/UNDP_GH_IG_GhanaCountryAnalysis2010_10102013.pdf.pdf (accessed on 2 March 2019).

22. Environmental Protection Agency. Ghana's Second National Communication (GSNC) to the UNFCCC; United Nations Development Programme: New York, NY, USA, 2011.

23. Blair, J.M.A. A climate for antibiotic resistance. Nat. Clim. Chang. 2018, 8, 460-461. [CrossRef]

24. MacFadden, D.R.; McGough, S.F.; Fisman, D.; Santillana, M.; Brownstein, J.S. Antibiotic Resistance Increases with Local Temperature. Nat. Clim. Chang. 2018, 8, 510-514. [CrossRef] [PubMed]

25. Alves, M.S.; Dias, R.C.D.S.; De Castro, A.C.D.; Riley, L.W.; Moreira, B.M. Identification of Clinical Isolates of Indole-Positive and Indole-Negative Klebsiella spp. J. Clin. Microbiol. 2006, 44, 3640-3646. [CrossRef] [PubMed]

26. CSLI. Performance Standards for Antimicrobial Susceptibility Testing; Clinical and Laboratory Standards Institute: Wayne, PA, USA, 2017. 
27. Sageerabanoo, S.; Malini, A.; Mangaiyarkarasi, T.; Hemalatha, G. Phenotypic detection of extended spectrum $\beta$-lactamase and Amp-C $\beta$-lactamase producing clinical isolates in a Tertiary Care Hospital: A preliminary study. J. Nat. Sci. Boil. Med. 2015, 6, 383-387.

28. Maraskolhe, D.; Deotale, V.; Mendiratta, D.; Narang, P. Comparision of Three Laboratory Tests for Detection of AmpC $\beta$ Lactamases in Klebsiella Species and E. Coli. J. Clin. Diagn. Res. 2014, 8, DC05-DC08. [PubMed]

29. Anwar, M.; Ejaz, H.; Zafar, A.; Hamid, H. Phenotypic Detection of Metallo-Beta-Lactamases in Carbapenem Resistant Acinetobacter baumannii Isolated from Pediatric Patients in Pakistan. J. Pathog. 2016, 2016, 1-6. [CrossRef] [PubMed]

30. Rasheed, J.K.; Anderson, G.J.; Yigit, H.; Queenan, A.M.; Doménech-Sánchez, A.; Swenson, J.M.; Tenover, F.C. Characterization of the Extended-Spectrum $\beta$-Lactamase Reference Strain, Klebsiella pneumoniae/K6 (ATCC 700603), Which Produces the Novel Enzyme SHV-18. Antimicrob. Agents Chemother. 2000, 44, 2382-2388. [CrossRef]

31. ElHariri, M.; Hamza, D.; Elhelw, R.; Dorgham, S.M. Extended-spectrum beta-lactamase-producing Pseudomonas aeruginosa in camel in Egypt: Potential human hazard. Ann. Clin. Microbiol. Antimicrob. 2017, 16, 710. [CrossRef]

32. Zhang, J.; Zheng, B.; Zhao, L.; Wei, Z.; Ji, J.; Li, L.; Xiao, Y. Nationwide high prevalence of CTX-M and an increase of CTX-M-55 in Escherichia coli isolated from patients with community-onset infections in Chinese county hospitals. BMC Infect. Dis. 2014, 14, 659. [CrossRef]

33. Fallah, F.; Noori, M.; Hashemi, A.; Goudarzi, H.; Karimi, A.; Erfanimanesh, S.; Alimehr, S. Prevalence of bla NDM, bla PER, bla VEB, bla IMP, and bla VIM Genes among Acinetobacter baumannii Isolated from Two Hospitals of Tehran, Iran. Sci. 2014, 2014, 1-6.

34. Mushi, M.F.; Mshana, S.E.; Imirzalioglu, C.; Bwanga, F. Carbapenemase Genes among Multidrug Resistant Gram Negative Clinical Isolates from a Tertiary Hospital in Mwanza, Tanzania. BioMed Res. Int. 2014, 2014, 1-6. [CrossRef] [PubMed]

35. Jia, W.; Li, C.; Zhang, H.; Li, G.; Liu, X.; Wei, J. Prevalence of Genes of OXA-23 Carbapenemase and AdeABC Efflux Pump Associated with Multidrug Resistance of Acinetobacter baumannii Isolates in the ICU of a Comprehensive Hospital of Northwestern China. Int. J. Environ. Res. Public Heal. 2015, 12, 10079-10092. [CrossRef] [PubMed]

36. Soudeiha, M.A.H.; Dahdouh, E.A.; Azar, E.; Sarkis, D.K.; Daoud, Z. In vitro Evaluation of the Colistin-Carbapenem Combination in Clinical Isolates of A. baumannii Using the Checkerboard, Etest, and Time-Kill Curve Techniques. Front. Microbiol. 2017, 7, 209.

37. Opintan, J.; Newman, M.J.; Arhin, R.E.; Donkor, E.S.; Gyansa-Lutterodt, M.; Mills-Pappoe, W. Laboratory-based nationwide surveillance of antimicrobial resistance in Ghana. Infect. Drug Resist. 2015, 8, 379-389. [CrossRef] [PubMed]

38. USAID. Ghana Climate Change Vulnerability and Adaptation Assessment. 2011. Available online: https://www.climatelinks.org/sites/default/files/asset/document/Climate\%20Change\%20Assessment_ Ghana_\%20FINAL.pdf (accessed on 2 March 2019).

39. Alividza, V.; Mariano, V.; Ahmad, R.; Charani, E.; Rawson, T.M.; Holmes, A.H.; Castro-Sánchez, E. Investigating the impact of poverty on colonization and infection with drug-resistant organisms in humans: A systematic review. Infect. Dis. Poverty 2018, 7, 76. [CrossRef] [PubMed]

40. Miller-Petrie, M.; Gelband, H. Socioeconomics, Antimicrobial Use and Antimicrobial Resistance. 2017. Available online: http://resistancecontrol.info/2017/socioeconomics-antimicrobial-use-and-antimicrobialresistance/ (accessed on 2 March 2019).

41. Codjoe, F.S. Detection and Characterisation of Carbapenem-Resistant Gram-Negative Bacilli Infections in Ghana; Sheffield Hallam University: Sheffield, UK, 2016. 
42. Ben Nasr, A.; Decré, D.; Compain, F.; Genel, N.; Barguellil, F.; Arlet, G. Emergence of NDM-1 in Association with OXA-48 in Klebsiella pneumoniae from Tunisia. Antimicrob. Agents Chemother. 2013, 57, 4089-4090. [CrossRef] [PubMed]

43. Robberts, F.J.L.; Kohner, P.C.; Patel, R. Unreliable Extended-Spectrum $\beta$-Lactamase Detection in the Presence of Plasmid-Mediated AmpC in Escherichia Coli Clinical Isolates. J. Clin. Microbiol. 2009, 47, 358-361. [CrossRef]

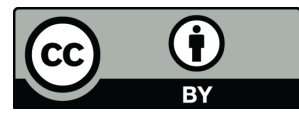

(C) 2019 by the authors. Licensee MDPI, Basel, Switzerland. This article is an open access article distributed under the terms and conditions of the Creative Commons Attribution (CC BY) license (http://creativecommons.org/licenses/by/4.0/). 